\title{
Mathematics and Teaching
}

\section{Hyman Bass}

*This article is reprinted, with permission, from the book I, Mathematician, edited by Peter Cassazza, Steven G. Krantz, and Randi D. Ruden, and published by the Mathematical Association of America, 2015.

\section{Induction}

I was not born to be a mathematician. Like many, I was drawn to mathematics by great teaching. Not that I was encouraged or mentored by supportive and caring teachers-such was not the case. It was instead that I had as teachers some remarkable mathematicians who made the highest expression of mathematical thinking visible and available to be appreciated. This was like listening to fine music with all of its beauty, charm, and sometimes magical surprise. Though not a musician, I felt that this practice of mathematical thinking was something I could pursue with great pleasure and capably so, even if not as a virtuoso. And I had the good fortune to be in a time and place where such pursuits were comfortably encouraged.

The watershed event for me was my freshman (honors) calculus course at Princeton. The course was directed by Emil Artin, with his graduate students John Tate and Serge Lang among its teaching assistants. It was essentially a Landau-style course in real analysis (i.e., one taught rigorously from first principles). Several notable mathematics research careers were launched by that course. Amid this cohort of brilliant students, I hardly entertained ideas of an illustrious mathematical future, but I reveled in this ambience of beautiful thinking, and I could think of nothing more satisfying than to remain a part of that world. It was only some fifteen years later that I came to realize that this had not been a more-or-less standard freshman calculus course.

Certain mathematical dispositions that were sown in that course remain with me to this day, and influence both my research and my teaching. First is the paramount importance of proofs as the defining source of mathematical truth. A theorem is a distilled product of a proof, but the proof is a mine from which much more may often be profitably extracted. Proof analysis may show that the argument in fact proves much more than the theorem statement captures. Certain hypotheses

Hyman Bass is professor of mathematics at the University of Michigan. His email address is hybass@umich . edu.

DOI: http://dx.doi.org/10.1090/noti1256 may not have been, or only weakly, used, and so a stronger conclusion might be drawn from the same argument. Two proofs may be observed to be structurally similar, and so the two theorems can be seen to be special cases of a more unifying claim. The most agreeable proofs explain rather than just establish truth. And the logical narrative clearly distinguishes the illuminating turn from technical routine.

Artin himself once reflected on teaching in a review published in 1953.

We all believe that mathematics is an art. The author of a book, the lecturer in a classroom tries to convey the structural beauty of mathematics to his readers, to his listeners. In this attempt, he must always fail. Mathematics is logical to be sure, each conclusion is drawn from previously derived statements. Yet the whole of it, the real piece of art, is not linear; worse than that, its perception should be instantaneous. We have all experienced on some rare occasion the feeling of elation in realising that we have enabled our listeners to see at a moment's glance the whole architecture and all its ramifications.

Two things of a more social character about mathematics also impressed me. First, the standards for competent and valid mathematical work appeared to be clear, objective, and (so I thought at the time) culturally neutral. The norms of mathematical rigor were for the most part universal and shared across the international mathematics community. Of course mathematical correctness is not the whole story; there is also the question of the interest and significance of a piece of mathematical work. On this score matters of taste and aesthetics come into play, but there is still, compared with other fields, a remarkable degree of consensus among mathematicians about such judgments. One circumstance that readily confers validation is a rigorous solution to a problem with high pedigree, meaning that it was posed long ago and has so far defied the efforts of several recognized mathematicians. 
A social expression of this culture of mathematical norms stood out to me. Success in mathematics was independent of outward trappings, physical or social, of the individual. This was in striking contrast with almost every other domain of human endeavor. People, for reasons of physical appearance, affect, or personality, were often less favored in nonmathematical contexts. But, provided that they met mathematical norms, such individuals would be embraced by the mathematics community. At least so it seemed to me, and this was a feature of the mathematical world that greatly appealed to me. I have since learned that, unfortunately, many mathematicians individually compromised this cultural neutrality, allowing prejudice to discourage the entry of women and other culturally defined groups into the field.

One effect of this intellectual indifference to social norms in mathematics is that a number of accomplished mathematicians do not present the socially favored images of appearance and/or personality, and so the field is sometimes caricatured as being one of brilliant but socially maladroit and quirky individuals. On the contrary, it is a field with the full range of personality types, and it is distinctive instead for its lack of the kind of exclusion based on personality or physical appearance that infects most other domains of human performance. Perhaps the intellectual elitism and sense of aristocracy common to many mathematicians act as a counterpoint to this social egalitarianism.

The second social aspect of mathematics that stood out to me concerned communication about mathematics to nonexperts. Throughout my student years, undergraduate and graduate, I was amazed and excited by the new horizons being opened up. I enthusiastically tried to communicate some of this excitement to my nonmathematical friends, from whom I had eagerly learned so much about their own studies. These efforts were increasingly frustrated despite my efforts to put matters in analytically elementary terms. I think perhaps that I had already become too much of a mathematical formalist and considered the formal rendering of the ideas an important part of the message. This rendering was often inaccessible to my friends despite my enthusiasm. As my research career entered more abstract theoretical domains, I gradually, and with disappointment, retreated somewhat from efforts to talk to others about what I did as a mathematician. Samuel Eilenberg, my mentor when I first joined the Columbia University faculty, once said something to the effect that

Mathematics is a performance art, but one whose only audience is fellow performers.

I remain to this day deeply interested in this communication problem, and I have admired and profited from the growing number of authors who have found the language, representations, styles, and narratives with which to communicate the nature of mathematics, its ideas, and its practices. Also, my current studies of mathematics teaching have reopened this question, but now in a somewhat different context. For twelve years of schooling, mathematics has a captive audience of young minds with a natural mathematical curiosity too often squandered. And these children's future teachers are students in the mathematics courses we teach.

\section{Mathematical Truth and Proof}

Each discipline has its notions of truth, norms for the nature and forms of allowable evidence, and warrants for claims. Mathematics has one of the oldest, most highly evolved, and well-articulated systems for certifying knowledge-deductive proof-dating from ancient Greece and eventually fully formalized in the twentieth century. There may be philosophical arguments about allowable rules of inference and about how generous an axiom base to admit, and there may be practical as well as philosophical issues about the production and verification of highly complex and lengthy, perhaps partly machine-executed, "proofs." But the underlying conceptual construct of (formal) proof is not seriously thrown into question by such productions, only whether some social or artifactual construction can be considered to legitimately support or constitute a proof.

Mathematical work generally progresses through a trajectory that I would describe as

$$
\begin{gathered}
\text { Exploration } \rightarrow \text { discovery } \rightarrow \text { conjecture } \\
\rightarrow \text { proof } \rightarrow \text { certification }
\end{gathered}
$$

In my work with Deborah Ball $(2000,2003)$ we have described the first three phases as involving reasoning of inquiry and the last two as reasoning of justification. The former is common to all fields of science. The latter has only a faint presence in mathematics education, even though it is the distinguishing characteristic of mathematics as a discipline.

Deductive proof accounts for a fundamental contrast between mathematics and the scientific disciplines: they honor very different epistemological gods. Mathematical knowledge tends much more to be cumulative. New mathematics builds on, but does not discard, what came before. The mathematical literature is extraordinarily stable and reliable. In science, by contrast, new observations or discoveries can invalidate previous models, which then lose their scientific currency. The contrast is sharpest in theoretical physics, which historically has been the science most closely allied with the development of mathematics. I. M. Singer is said to have once compared the theoretical physics literature to a blackboard that 
must be periodically erased. Some theoretical physicists-Richard Feynman, for instance-enjoyed chiding the mathematicians' fastidiousness about rigorous proofs. For the physicist, if a mathematical argument is not rigorously sound but nonetheless leads to predictions that are in excellent conformity with experimental observation, then the physicist considers the claim validated by nature, if not by mathematical logic-nature is the appropriate authority. The physicist P. W. Anderson once remarked, "We are talking here about theoretical physics, and therefore of course mathematical rigor is irrelevant."

On the other hand, some mathematicians have shown a corresponding disdain for this freewheeling approach of the theoretical physicists. The mathematician E. J. McShane once likened the reasoning in a "physical argument" to that of "the man who could trace his ancestry to William the Conqueror, with only two gaps."

Even some mathematicians eschew heavy emphasis on rigorous proof in favor of more intuitive and heuristic thinking and of the role of mathematics to help explain the world in useful or illuminating ways. In general they do not necessarily scorn rigorous proof, only consign it to a faintly heeded intellectual super-ego. But I would venture nonetheless, that, for most research mathematicians, the notion of proof and its quest are at least tacitly central to their thinking and their practice as mathematicians. And this would apply even to mathematicians who, like Bill Thurston, view what mathematicians do as not so much the production of proofs, but as "advancing mathematical understanding" (Thurston, 1994). It would be hard to find anyone with the kind of mathematical understanding and function of which Thurston speaks who has not already assimilated the nature and significance of mathematical proof.

At the same time, the writing of rigorous mathematical proofs is not the work that mathematicians actually do, for the most part, or what they most cherish and celebrate. Such tributes are conferred instead on acts of creativity, of deep intuitive discovery, of insightful analysis and synthesis. André Weil (1971) said,

If logic is the hygiene of the mathematician, it is not his source of food; the great problems furnish the daily bread on which he thrives.

Vladimir Arnold offered an even more derisory characterization:

Proofs are to mathematics what spelling (or even calligraphy) is to poetry. Mathematical works do consist of proofs, just as poems do consist of characters.
But it is proof that finally gives mathematical achievements their pedigree.

\section{The Proof vs. Proving Paradox}

Saying that a mathematical claim is true means, for a mathematician, that there exists a proof of it. Strictly speaking, this is a theoretical concept, independent of any physical artifact and therefore also of any human agency. But proving is an undeniably human activity and so susceptible to human fallibility. It is an act of producing conviction about the truth of something. A mathematician, in proving a mathematical claim, will typically produce a manuscript purporting to represent a mathematical proof and exhibit it for critical examination by expert peers (certification). But as Lakatos (1976) has vividly described, this process of proof certification can be errant and uneven, though ultimately robust.

The rules for proof construction are sufficiently exact that the checking of a proof should, in principle, be a straightforward and unambiguous procedure. However, formal mathematical proofs are ponderous and unwieldy constructs. For mathematical claims of substantial complexity, mathematicians virtually never produce complete formal proofs. Indeed, requiring that they do so would cause the whole enterprise to grind to a halt. The resulting license in the practices of certifying mathematical knowledge has caused some (nonmathematical) observers to conclude that mathematical truth is just another kind of social negotiation and, so, unworthy of its prideful claims of objective certainty.

I think that this notion is based on a misunderstanding of what mathematicians are doing when they claim to be proving something. Specifically, I suggest that

Proving a claim is, for a mathematician, an act of producing, for an audience of peer experts, an argument to convince them that a proof of the claim exists.

Two things are important to note here. First, that implicit in this description is the understanding that the peer experts possess the conceptual knowledge of the nature and significance of mathematical proof. Second, the notion of "conviction" here is operationalized to mean that

The convinced listener feels empowered by the argument, given sufficient time, resources, and incentive, to actually construct a formal proof.

Of course, in this time of computer-aided proofs, some of this certification must be transferred to establishing the reliability of proof-checking software.

In my work with Deborah Ball, we have found this perspective helpful in studying the develop- 
ment of what might reasonably be called mathematical proving in the early grades. I say more about this development below.

\section{Compression and Abstraction}

Mathematical knowledge is, as I mentioned above, cumulative (nothing is discarded) and also hierarchical. What saves it then from sinking under the weight of its own relentless growth into some dense impenetrable massive network of ideas? It is rescued from this by a distinctive feature of mathematics that some have called compression. This is a process by which certain fundamental mathematical concepts or structures are characterized and named and so cognitively rescaled so that they become, for the expertly initiated, as mentally manipulable as counting numbers is for a child. Think for example what a complex of ideas (and mathematical history) is packed into an expression like "complex Lie group," uttered fluently among mathematicians. Think of the years of schooling needed to invest that expression with precise meaning.

A typical form of compression arises from the unification of diverse phenomena as special cases of a single construct (for example, groups, topological spaces, Hilbert spaces, measure spaces, categories and functors, etc.) about which enough of substance can be said in general to constitute a useful unifying theory. This leads to another salient feature of mathematics: abstraction. Most mathematics has its roots in science and so ultimately in the "real world." But mathematics, even that contrived to solve real-world problems, naturally generates its own problems, and so the process continues with these, leading to successive stages of unification through abstraction until the mathematics may be several degrees removed from its empirical origins. It is a happy miracle that this process of pursuing natural mathematical questions repeatedly reconnects with empirical reality in unexpected and unplanned ways. This is the "unreasonable effectiveness" of which Wigner wrote (1960) and of which Varadarajan (2015) writes eloquently in "this collection of essays.

Abstraction is often thought to separate mathematics from science. But even among mathematicians there are different professed dispositions toward abstraction. Some mathematicians protest that they like to keep things "concrete," but a bit of reflection on what they consider to be concrete will show it to be far from such for an earlier generation. Indeed, I noticed while a graduate student that the extent to which a mathematical idea was considered abstract seemed more a measure of the mathematician's age than of the cognitive nature of the idea. As new ideas become assimilated into courses of instruction, they become the daily bread and butter of initiates, all the while remaining novel and exotic to many of their elders.
While compression is an essential instrument for the ecological survival of mathematics, its very virtue presents a serious obstacle for the teaching of mathematics. The knowledgeable and skillful mathematician has assimilated and internalized years of successive compression, streamlining of ideas, and habits of mind. But a young learner of mathematics still lives and thinks in a "mathematically decompressed" world, one that has become hard for the mathematically trained person to imagine, much less remember. This presents a special challenge to teachers of mathematics to children. And, interestingly, it requires a special kind of knowledge of mathematics itself which is neither easy for nor common among otherwise mathematically knowledgeable adults. (See Ball et al., 2005, 2008.)

\section{Teaching Mathematics}

Among the questions to which our editors invited us to direct attention was, "How are we research mathematicians viewed by others?" For one community, (school) mathematics teachers and education researchers, I have some firsthand knowledge of this after more than a decade of interdisciplinary work in mathematics education, and what I have learned I find both interesting and important for mathematicians to understand. I began this essay with an account of how great university teaching drew me into mathematics. Now, in my close study of elementary mathematics teaching, I have a changed vision of what teaching entails.

Of course many mathematicians, like mathematics educators, are seriously interested in the mathematics education of students. But there are significant differences in how the two communities, broadly speaking, see this enterprise. The mathematicians' interests, naturally enough, are directed primarily at the graduate and undergraduate levels and perhaps somewhat at the secondary level. In contrast, the interests of the educators are predominantly aimed at the primary through secondary levels. But there is a broadening overlap in the ranges of interest of the two communities, and some fundamental aspects of what constitutes quality teaching are arguably independent of educational levels. Nonetheless, in areas of common focus there are often profound differences of perspective and understanding across the two communities about what constitutes quality mathematics instruction. I expand on this below.

There is also a difference in educational priorities between the mathematics and mathematics education communities, a difference whose importance cannot be overstated. Mathematicians are naturally interested in "pipeline" issues, the rejuvenation of the professional community, and so the induction and nurturing of talented and highly motivated students into high-level mathematical study. Mathematics educators, on the 
other hand, are professionally committed to the improvement of public education at scale, with the aim of high levels of learning for all students and with a heavy emphasis on the word "all." While these two agendas are not intrinsically in conflict or competition, they are often seen to be so, and this can have resource and policy implications. Public education in the US, despite long and costly interventions, continues to perform poorly in international comparisons in terms of meeting workplace demands and even in providing basic literacies. Moreover, there is a persistent achievement gap reflected in underperformance of certain subpopulations that the educational system historically has not served well. These are the "big frontline problems" of mathematics education, and they are just as compelling and urgent to mathematics educators as the big research problems are to mathematicians.

Mathematicians have an excellent tradition of nurturing students of talent. What they are less good at is identifying potential talent. The usual indices, high test scores and precocious accomplishment, are easy enough to apply, but these will typically overlook students of mathematical promise whom the system has not encouraged or given either the expectation of or opportunity for high performance. As a result, mathematical enrichment programs, if not sensitively designed, can sometimes perpetuate the very inequities that mathematics educators are trying to mitigate.

My main focus here, however, is on teaching. Much of what I have learned I owe to work with my colleague and collaborator, Deborah Ball. To facilitate what I want to say, it will be helpful to use a schematic developed by Deborah and her colleagues David K. Cohen and Stephen W. Raudenbush (2003) to describe the nature of instruction.

\section{The "Instructional Triangle"}

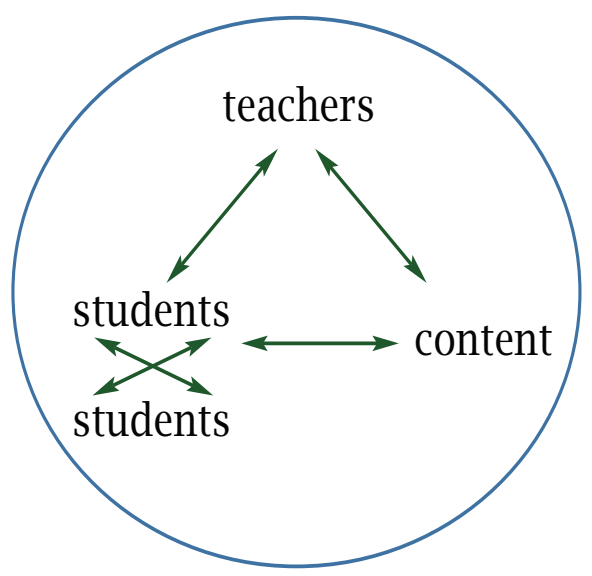

The concept proposed by this image is that instruction is about the interactions of the teacher with students (and of students with each other) around content. The double arrows emphasize that these interactions are dynamic; in particular, changing any one element of the picture significantly alters the whole picture.

Now what I suggest is that most mathematicians' conception of instruction lives primarily at the content corner of the triangle, with its school incarnation expressed in terms of curriculum (including both standards documents and curriculum materials). In this view, with high-quality curriculum in place, the mathematically competent teacher has only to implement that curriculum with intellectual fidelity, and then attentive and motivated students will learn. Of course mathematicians have little direct influence on schoolchildren, but they often have explicit, disciplineinspired ideas about what mathematics children should learn and how. In the case of teachers, mathematicians do bear some direct responsibility for their mathematical proficiency, since many teachers learn much of the mathematics they know in university mathematics courses. Many studies have pointed to weak teacher content knowledge as a major source of underperformance in public education. Of the many remedies proposed, mathematicians have generally favored more and higher-level mathematics courses as a requirement for certification. Unfortunately, none of the interventions based on these ideas so far undertaken have yet produced the desired gains in student achievement for a broad range of students. Note that all of this discussion and debate resides on the content-teacher side of the triangle, with little explicit attention yet given to the role of the students in instruction. Effective teachers teach both math and children.

An educational counterpart to the above "onesided" (teacher-content) stance is an intellectually adventurous, but somewhat romantic, view of school teaching, this time on the teacher-student side of the triangle. This approach proposes offering tangible and somehow "real-world" related mathematical activities with which to engage learners but leaving the development of mathematical ideas to largely unrestrained student imagination and invention. In such cases the discipline of the mathematical ideas may be softened to the point of dissipation.

The most refined understanding of mathematics teaching, of which Deborah Ball's work is exemplary, insists that teaching must coordinate attention to the integrity of the mathematics and appropriate learning goals with attention to student thinking, which needs to be honored and made an integral part of the instruction. In this view, the effective teacher has not only a deep understanding of and fascination with the mathematics being taught but also a dual knowledge of and fascination with student thinking. The underlying premise is that children have significant mathematical ideas, albeit imperfectly 
expressed, and that a part of the teacher's work is to recognize the presence of those ideas (for which a sophisticated knowledge of mathematics is needed!), to give them appropriate validation, and to help students shape them into a more developed articulation and understanding. It is the coordination of these dual spheres of attention, both to the mathematics and to students' thinking, that makes effective teaching the intricate and skilled work that it is.

This is a kind of professional practice that discursive rhetoric cannot adequately capture. It is best conveyed through an examination of teaching practice itself. So let me offer a vignette. Consider the teaching of mathematical proving. This is typically first done in high school geometry, but there are good reasons to argue that it should be done developmentally, starting in the early grades. What might this mean or look like? After all, young children have no concept of anything approaching mathematical proof, and no one seriously argues that this should be formally taught to them. First of all, what is the intellectual imperative for proving that can be made meaningful to young children? Our view is that it is the persistent question, "Why are things true?" Deductive proof is the refined method devised by mathematicians to answer that very question. Learning the methods of deductive proving takes time, but the question Why are things true? is itself immediately compelling. The underlying pedagogy is that students progressively learn the methods of constructing compelling mathematical arguments in the course of repeatedly trying to convince others of things they have good reasons to believe to be true. In other words, students can be helped to construct the infrastructure of proving in the very course of proving things (to the best of their growing abilities).

To illustrate this idea, I turn to an episode from a third-grade class (8-year-olds). The children have been exploring even and odd numbers. Although they do not yet have formal definitions, they rely on intuitive ideas of even and odd based on notions of fair-sharing, and they can identify whether particular (small) numbers are even or odd. They begin to notice addition patterns, like: even + even $=$ even, even + odd $=$ odd, and odd + odd $=$ even. The teacher asks them whether they can "prove" that "Betsy's Conjecture" (odd + odd = even) is always true. Some of the children have tested the conjecture with lots of numbers, and they are thereby convinced that it must be true. The teacher challenges them, "How do you know that someone might not come up with a new example that doesn't work?" The children are left to ponder that, some of them working collaboratively in small groups.

The next day, the teacher asks the class what they found out. Several hands go up, and she calls on Jeannie, who, speaking also for her partner,
Sheena, says, "We were trying to prove that...you can't prove that Betsy's Conjecture always works... because numbers go on and on forever, and that means odd numbers and even numbers go on forever and, um, so you couldn't prove that all of them work." This stunning assertion is at once insightful and revolutionary. Jeannie's comment reflects the fact that Betsy's Conjecture is not just one, but rather infinitely many claims, and so not susceptible to empirical verification. Though articulated somewhat informally, she has realized that the cases of the conjecture go on and on.

A second student, Mei, objects to this argument. She objects not to the reasoning but rather to an important inconsistency. She points out that prior claims for which the class had achieved consensus were also not checked in all cases, and Jeannie and her partner had not raised any similar objection to those earlier claims. She challenges the need to make sure that the claim works for all cases, since they had not tried to do this for other conjectures.

In both of these contributions, one can see the process by which the class is beginning to construct the very norms of mathematical reasoning: rejecting empirical methods for an infinitely quantified claim in the first instance and the need for consistency of logical methods in the second. And all of this is still short of proving Betsy's Conjecture.

But the next day, Betsy produces a "proof" of her conjecture. At the board she draws a row of seven small circles and then to the right another row of seven small circles. Then she partitions the two rows into three groups of two, with one left over, and the ones left over next to each other in the middle:

$$
\underline{0 O} \underline{0 O} \underline{0 O} O \quad 0 \underline{O O} \underline{O O} \underline{0 O}
$$

Then she turns to the class and explains haltingly, "If you have an odd number and group it by twos you have one left over, so if you have two odd numbers and group them by twos, then the ones left over can be grouped together so you have an even number." What is most significant about this oral argument is she makes no reference to the specific numbers 7 and 7 that she has drawn, but rather speaks generically of "an odd number."

How does this demonstration surmount the dilemma raised by Jeannie and Sheena? Betsy has implicitly invoked a general definition of odd number-a number that when grouped by twos has one left over-and this definition, being itself infinitely quantified (it characterizes all odd numbers), can support a conclusion of similarly infinite purview. Betsy's oral argument might be algebraically expressed in the form

$$
\left(x_{2}+1\right)+\left(1+y_{2}\right)=x_{2}+(1+1)+y_{2}=(x+1+y)_{2} .
$$

(Note that, for children at this stage, $2 x=x+x$ is not the same as $x_{2}=2+2+\cdots+2$.) But, although she 
is thinking in general terms, Betsy does not yet know how to represent her ideas generally using algebraic notation.

Notice for each of these students-Jeannie, Mei, and Betsy-the significance of the mathematical ideas, albeit sometimes expressed with difficulty. Episodes like this demonstrate what it might look like to develop the norms of proving in the course of constructing more and more well-developed arguments to convince others of the truth of claims. But this is not the whole story. This narrative resides, as does much of the education research literature, on the student-content side of the instructional triangle, focusing on accomplished student performance. The teacher seems invisible.

The above kind of collective interaction and learning does not occur spontaneously, unmediated by well-informed and purposeful instruction. The question of what makes for effective teaching has to do with understanding the knowledge, skills, and sensibilities (both mathematical and pedagogical) and, above all, the practices that enable instruction that can elicit the kind of motivation and reflective engagement of students described in the episode above. The kind of mathematical knowledge required includes not only a robust understanding of the mathematical terrain of the work and corresponding learning goals but also an ability to hear, in incipient and undeveloped form, significant, though often not entirely correct, mathematical ideas in student thinking. I emphasize that the latter entails a special kind of knowledge of mathematics, not just psychology. Teaching requires the skills to not only hear and validate and give space to these ideas, but also to help students reshape them in mathematically productive ways. The teacher needs to know how to instruct with questions more than with answers and to give students appropriate time, space, and resources to engage these questions. The actions to accomplish this, though purposeful, structured, and carefully calibrated to the students, are also subtle, deliberately focusing on student performance. And so, to the naive observer, it often can appear that "the teacher is not doing much." The central problem of teacher education is to provide teachers with the knowledge and skills of such effective practice.

The idea of developmental learning, such as we saw with the third-graders above, was also a feature of my calculus course back at Princeton in 1951. I first learned in that course what (a strange thing) a real number was (mathematically), ${ }^{1}$ even though I had been working comfortably with real numbers throughout the upper high school grades. With regard to proving, while we learned, through witnessing stunning examples and through practice problems how to construct reasonably

${ }^{1}$ An equivalence class of Cauchy sequences of rational numbers. rigorous proofs, it was still always the case that the claims in question seemed at least reasonable and sufficiently meaningful that general mathematical intuition could guide us. But I was at first stymied by a problem on one of our take-home exams. A function $f(x)$ was defined by: $f(x)=0$ for $x$ irrational, and $f(\mathrm{x})=1 / q$ if $x$ is rational, equal to $p / q$ in reduced form. The question was, "Where is $f$ continuous?" At first sight this question seemed outrageous. How could one possibly answer it? It was impossible to sketch the graph. Intuition was useless. After some reflection I resigned myself to the fact that all we had to work with were definitions of $f$ and of continuity. It was a great revelation to me that these definitions and a modest amount of numerical intuition sufficed to answer the question. This was a great lesson about the nature and the power of deductive reasoning, to which my enculturation, though at a different level, was not so different from that of the third-graders above.

The construction and timing of such a problem was itself a piece of instruction. I wish now that we had video records of Emil Artin's calculus teaching that I could show to an accomplished teacher, like Deborah, to analyze the pedagogical moves, including interaction with students, of which his practice was composed. Much as I profited from that instruction, I was not prepared to see the craft of its construction.

\section{References}

D. L. BALL and H. BASS, Making believe: The collective construction of public mathematical knowledge in the elementary classroom. D. Philips (ed.), Constructivism in education: Yearbook of the National Society for the Study of Education, University of Chicago Press, Chicago, 2000, pp. 193-224.

D. L. BALL, H. C. HILL, and H. BASs, Knowing mathematics for teaching: Who knows mathematics well enough to teach third grade, and how can we decide? American Educator 29(1) (2005), 14-17, 20-22, 43-46.

D. L. BALl, M. H. Thames, and G. PhelPS, Content knowledge for teaching: What makes it special? Journal of Teacher Education 59(5) (2008), 389-407.

D. K. Cohen, S. W. Raudenbush, and D. L. BALl, Resources, instruction, and research, Educational Evaluation and Policy Analysis 25(2) (2003), 119-142.

I. LAKATOS, Proofs and Refutations: The Logic of Math ematical Discovery, Cambridge University Press, 1976.

WILLIAM P. THURSTON, On proof and progress in mathematics, Bull. Amer. Math. Soc. (N.S.) 30(2) (1994), 161-177.

V. S. VARADARAJAN, "I am a mathematician" (essay), I, Mathematician. P. Cassazza, S. G. Krantz, and R. D. Ruden (eds.), Mathematical Association of America, 2015.

ANDRÉ WEIL, The future of mathematics, Great Currents of Mathematical Thought, Vol. 1, F. Le Lionnais (ed.), Dover Publications, New York, 1971, pp. 321-336.

EUGENE WIGNER, The unreasonable effectiveness of mathematics in the natural sciences, Comm. Pure Appl. Math. 13(1) (1960), 1-14. 